\title{
Revisiting the influence of unidentified binaries on velocity dispersion measurements in ultra-faint stellar systems
}

\author{
Alan W. McConnachie \\ alan.mcconnachie@nrc-cnrc.gc.ca \\ Patrick Côté \\ patrick.cote@nrc-cnrc.gc.ca \\ NRC Herzberg Institute of Astrophysics, 5071 West Saanich Road, Victoria, B.C., V9E \\ 2E7, Canada
}

Received —

Submitted to ApJL, March 292010 


\begin{abstract}
Velocity dispersion measurements of recently discovered Milky Way satellites with $M_{V} \gtrsim-7$ imply they posses high mass-to-light ratios. The expected velocity dispersions due to their baryonic mass are $\sim 0.2 \mathrm{~km} \mathrm{~s}^{-1}$, but values $\gtrsim 3 \mathrm{~km} \mathrm{~s}^{-1}$ are measured. We perform Monte Carlo simulations of mock radial velocity measurements of these systems assuming they have mass-to-light ratios similar to globular clusters and posses an unidentified binary star population, to determine if these stars could boost the velocity dispersion to the observed values. We find that this hypothesis is unlikely to produce dispersions much in excess of $\sim 4.5 \mathrm{~km} \mathrm{~s}^{-1}$, in agreement with previous work. However, for the systems with potentially the smallest velocity dispersions, values consistent with observations are produced in $5-40 \%$ of our simulations for binary fractions in excess of $f_{\text {bin }}(P \leq 10 \mathrm{yrs}) \sim 5 \%$. This sample includes the dwarf galaxy candidates that lie closest to classical globular clusters in $M_{V}-r_{h}$ space. Considered as a population, it is unlikely that all of these dwarf galaxy candidates have mass-to-light ratios typical of globular clusters, but boosting of the observed dispersion by binaries from near-zero values cannot be ruled out at high confidence for several individual dwarf galaxy candidates. Given the importance of obtaining accurate velocity dispersions and dynamical masses for the faintest satellites, it is clearly desirable to exclude directly the possible effect of binaries on these systems. This requires multi-epoch radial velocity measurements with individual uncertainties of $\lesssim 1 \mathrm{~km} \mathrm{~s}^{-1}$ to identify spectroscopic binaries with orbital velocities of order the observed velocity dispersion.
\end{abstract}

Subject headings: binaries: general — galaxies: dwarf — galaxies: kinematics and dynamics — galaxies: star clusters: general — galaxies: structure — Local Group 


\section{Introduction}

The internal velocity dispersions of Milky Way (MW) dwarf galaxies with $M_{V} \leq-8$ lie in the range $6<\sigma_{o b s}<11 \mathrm{~km} \mathrm{~s}^{-1}$ (Aaronson 1983). These values are large given the observed luminosities and surface brightnesses, and prompted early research to consider unidentified spectroscopic binary stars as a potential explanation for the unexpectedly high velocity dispersions (e.g., Mateo et al. 1993; Olszewski et al. 1996; Hargreaves et al. 1996). If many of the targeted stars are binaries, then the radial velocity that is measured is a superposition of their intrinsic radial velocity within the galaxy and their binary orbital velocity. Simulations of plausible binary populations showed that, while some inflation of the dispersion may be expected due to binaries, this effect is generally insufficient to explain the high velocity dispersions that are measured for the dwarf galaxies. More recently, Minor et al. (2010) develop a methodology to determine the contribution to the velocity dispersion of a dwarf galaxy from a binary population with a given set of parameters, for a well sampled dataset.

Here we revisit the role of binary stars in boosting the observed velocity dispersion of intrinsically low-mass systems. We are motivated by the discoveries of MW satellites with considerably lower luminosity $\left(M_{V} \gtrsim-7\right)$ than previously known dwarf galaxies, a regime that was not considered in the original papers on this subject. Some of these systems have few bright stars suitable for spectroscopic follow-up, and a few have measured velocity dispersions as low as $\sigma_{\text {obs }} \sim 3 \mathrm{~km} \mathrm{~s}^{-1}$ (implying mass-to-light ratios $\Upsilon_{V} \equiv M / L_{V} \gtrsim 100$ ). In Section 2, we summarize the relevant properties of these satellites, and we discuss how we generate realistic binary populations. In Section 3, we use Monte-Carlo simulations to investigate the effect of binary stars on velocity dispersion measurements of these systems assuming they have a near-zero intrinsic dispersion. 


\section{Preliminaries}

\subsection{The faint population of Milky Way satellites}

Fourteen new MW satellites discovered since 2004 (Willman et al.|2005; Willman et al. 2006; Zucker et al. 2006a.b; Belokurov et al. 2006, 2007, 2008, 2009; Sakamoto \& Hasegawa 2006; Irwin et al. 2007; Walsh et al. 2007) have been shown to possess velocity dispersions significantly higher than expected if they have mass-to-light ratios typical of globular clusters in virial equilibrium 1 (Martin et al. 2007; Simon \& Geha 2007; Geha et al. 2009; Koch et al. 2009; Walker et al. 2009; Adén et al. 2009; Belokurov et al. 2009). Their luminosities span more than five magnitudes, with the brightest systems (Canes Venatici I and Leo T) of comparable luminosity to Draco (the faintest MW dwarf galaxy known previously).

Table 1 summarizes the relevant structural and kinematic data for recently discovered faint MW satellites. We define this as $M_{V} \gtrsim-7$, and note that this sample includes three systems (Ursa Major I, II and Bootes I) with velocity dispersions of the same order as those of previously known dwarfs. In addition to the observed velocity dispersion $\left(\sigma_{o b s}\right)$, we list the number of putative member stars each measurement is based on $\left(n_{\text {stars }}\right)$, and the median velocity uncertainty $\left(\tilde{\mathrm{v}}_{\text {err }}\right)$ of those members. Leo $\mathrm{V}$ has two dispersion measurements, a lower central value using five stars and a larger global value using an additional two stars located at larger radius. Here, we quote the larger value. Further, Walker et al. (2009) do not rule out that Leo $\mathrm{V}$ may be a star cluster since its dispersion is presently barely resolved. We do not include Bootes III $\left(\sigma_{o b s} \simeq 14 \mathrm{~km} \mathrm{~s}^{-1}\right)$ in Table 1; Grillmair (2009) and Carlin et al. (2009) both suggest that Bootes III is a dwarf galaxy undergoing tidal

\footnotetext{
${ }^{1}$ Belokurov et al. (2010) recently discovered Pisces II, a candidate MW dwarf galaxy that does not yet have a velocity dispersion measurement.
} 
disruption. While we confine our analysis to the MW satellite population, we note that a similar analysis can also be applied to other Local Group satellites, such as the M31 satellite system, for which kinematic data is now becoming available (e.g., Kalirai et al. 2010; Collins et al. 2010).

For each satellite in Table 1, we calculate what their properties would be if they possess structural and kinematic characteristics typical for globular clusters (whose mass-to-light ratios are consistent with expectations from population synthesis models, without dark matter). Specifically, we derive their concentration, $c$, and core radius, $r_{c}$ using the relations of McLaughlin (2000), and derive the corresponding intrinsic velocity dispersion, $\sigma_{i n t}$, using

$$
\sigma_{i n t}=\left(\frac{2 \pi G \alpha p M}{9 \nu r_{c}}\right)^{\frac{1}{2}}
$$

assuming $M=\Upsilon_{V} L_{V}$ where $\Upsilon_{V}=1.45 \pm 0.1$ in solar units (McLaughlin 2000). The constants $\nu, \alpha$ and $p$ are calculated by interpolation of the values given in King (1966) and Peterson \& King (1975). Results are shown in Table 2. The uncertainty on $\sigma_{\text {int }}$ corresponds to the uncertainty on $\Upsilon_{V}$. The derived values for $\sigma_{\text {int }}$ are $\sim 0.2 \mathrm{~km} \mathrm{~s}^{-1}$, generally at least an order of magnitude smaller than $\sigma_{o b s}$ and $\tilde{\mathrm{v}}_{e r r}$.

\subsection{Calculating the velocity dispersion}

We will derive velocity dispersion estimates from mock radial velocity catalogs for these satellites by attempting to reproduce the observational techniques used to measure $\sigma_{o b s}$. In general, this involves identifying stars belonging to the system and measuring their mean velocity and dispersion. Here, we know a priori that our stars are members, but we will not necessarily use all the stars in our mock catalog to measure the dispersion. For example, some short-period binaries may have very different velocities from the mean of the 
sample, and they may not be observationally identified as members of the satellite. Instead, we sigma-clip our samples at $3 \sigma$ in order to mimic observations as closely as possible (e.g., Simon \& Geha 2007; Martin et al. 2007). At most, this removes only 1 or 2 stars from the final sample. All of the putative member stars identified in the actual observations summarized in Table 1 lie within $3 \sigma$ of the mean velocity, and so this seems reasonable.

Several of the radial velocity studies listed in Table 1 calculate the velocity dispersion $\left(\sigma_{P}\right)$ and mean velocity $(\bar{u})$ of the putative members by maximizing the natural logarithm of the probability function,

$$
\begin{aligned}
\ln (p)= & -\frac{1}{2} \sum_{i=1}^{n_{\text {star }}} \ln \left(v_{i, \text { err }}^{2}+\sigma_{p}^{2}\right)-\frac{1}{2} \sum_{i=1}^{n_{\text {star }}} \frac{\left(v_{i}-\bar{u}\right)^{2}}{\left(v_{i, \text { err }}^{2}+\sigma_{p}^{2}\right)} \\
& -\frac{n_{\text {stars }}}{2} \ln (2 \pi)
\end{aligned}
$$

(Walker et al. 2006), where $v_{i, e r r}$ is the measurement uncertainty on the measured velocity, $v_{i}$, of the $i^{\text {th }}$ star. We use this technique to calculate the velocity dispersion of our mock datasets for every satellite. We test this method on the observed datasets, and derive the same central values as the published results, with the exception of Segue II and Leo V. For Segue II, Belokurov et al. (2009) use a different likelihood analysis; for Leo V, Walker et al. (2009) use a similar technique to Equation 2 but incorporate the probability that a star is a member of the system. To ensure consistency, we recalculate the velocity dispersions for Segue II and Leo V using Equation 2 and use these new values in our subsequent analysis. These are included in Table 1 in parentheses, with the uncertainties calculated following Walker et al. (2006). 


\subsection{The binary star population}

The observed radial velocity of the primary companion in a binary star system is given by

$$
V_{r}=\frac{2 \pi a_{1} \sin i}{P\left(1-e^{2}\right)^{\frac{1}{2}}}[\cos (\theta+\omega)+e \cos \omega] .
$$

$a_{1}$ corresponds to the semi-major axis of the relative orbit of the primary; $P$ is the period of the orbit; $e$ is the eccentricity; $i$ is the orbital inclination to our line-of-sight; $\theta$ is the phase of the binary measured from periastron; $\omega$ is the longitude of periastron; $m_{1}$ and $m_{2}$ are the masses of the primary and secondary, respectively. $m=m_{1}+m_{2}$ and the mass ratio $q=m_{2} / m_{1}$. For an equal-mass binary system on a circular orbit with $m=1 M_{\odot}$ and $P=1$ yr, the maximum radial velocity that can be observed is $V_{r, \text { max }} \simeq \pm 15 \mathrm{~km} \mathrm{~s}^{-1}$. For $P=[10,100,1000] \mathrm{yrs}, V_{r, \max } \simeq[ \pm 7,3,1.5] \mathrm{km} \mathrm{s}^{-1}$.

We now summarize the adopted distributions for each of the variables in Equation 3. At the present time, the relevant physical parameters are entirely unknown for the stellar systems under discussion, and so we must be guided in our choice by the findings from other stellar populations, particularly the solar neighborhood (SN) and halo globular clusters.

\subsubsection{Mass distributions}

We set the mass of the primary to be $m_{1}=0.8 M_{\odot}$; this is the approximate minimum mass of a star that has had time to evolve on to the giant branch. The studies summarised in Table 1 target either giants or stars near the main-sequence turn-off, and so this seems reasonable. 
Duquennoy \& Mavor (1991) show that the mass ratio distribution for binary stars in the $\mathrm{SN}$ is well approximated as log-normal,

$$
\frac{d N}{d q} \propto \exp \left[-\frac{(q-\bar{q})^{2}}{2 \sigma_{q}^{2}}\right]
$$

$\bar{q}=0.23$ and $\sigma_{q}=0.42$. We set $q_{\min }=0.1$, so that the minimum mass of the secondary approximately corresponds to the limiting mass for hydrogen burning.

\subsubsection{Period distribution}

We use two different binary period distributions. We first follow Duquennoy \& Mavor (1991), who show that the distribution for SN binaries is described by a log-normal,

$$
\frac{d N}{d \log _{10} P} \propto \exp \left[-\frac{\left(\log _{10} P-\overline{\log _{10} P}\right)^{2}}{2 \sigma_{\log _{10} P}^{2}}\right] .
$$

$\overline{\log _{10} P}=4.8$ and $\sigma_{\log _{10} P}=2.3$, where $P$ is measured in days. We also explore a uniform distribution in $\log _{10} P$.

The maximum possible period of a binary star in a stellar cluster, $P_{\max }$, is set physically by the maximum separation beyond which the binary becomes unbound due to stellar interactions within the cluster. Hills (1984) derive the semi-major axis at which this occurs,

$$
a_{\max }=12.4 A U\left(\frac{m_{1}+m_{2}}{1.4 M_{\odot}}\right)\left(\frac{10 k m s^{-1}}{\sigma}\right)^{2}
$$

where $\sigma$ corresponds to the intrinsic velocity dispersion of the cluster. For the values of $\sigma_{\text {int }}$ in Table 2, this corresponds to $P_{\max } \sim 10^{7} \mathrm{yrs}$, or $V_{r, \max } \sim 0.14 \mathrm{~km} \mathrm{~s}^{-1}$, of order $\sigma_{\text {int }}$. However, such systems, if present, are likely very rare (following Equation 5) and cannot 
contribute to inflating the velocity dispersion by more than a small fraction of a $\mathrm{km} \mathrm{s}^{-1}$.

Constraints on $P_{\min }$ can be derived physically for red giant branch (RGB) stars from mass transfer considerations,

$$
a_{\text {min }}=\frac{R_{1}}{h(q)}, \quad 0.38 \lesssim h(q) \lesssim 0.59
$$

where $R_{1}$ is the radius of a star on the RGB and $h(q)$ is a slowly varying function that gives the volume radius of the Roche lobe in terms of the orbital separation (see $\S \mathrm{V}$ of Pryor et al. 1988). In old, metal-poor populations, luminous RGB stars have $R_{1} \sim 0.5 \mathrm{AU}$,

and so we conservatively adopt $\left\langle a_{\text {min }}\right\rangle \sim 1$ AU. Thus, $P_{\text {min }} \sim 1$ yr typically, although values as low as $0.1 \mathrm{yr}$ are plausible, particularly for stars fainter than the tip of the RGB.

\subsubsection{Eccentricity distribution}

Two eccentricity distributions are used. Following Heggie (1975), we first assume a thermal distribution in eccentricity,

$$
\frac{d N}{d e} \propto 2 e
$$

In the second instance, we assume circular orbits throughout $(e=0)$

\subsubsection{Angles}

The probability of observing a binary system at an inclination $i$ is proportional to sin $i$. The probability of observing a binary system with a given orientation of its major axis to the line of sight, $\omega$, is uniform between 0 and $\pi$. The probability of observing a 
binary system at a given phase, $\theta$, is proportional to the inverse of the angular velocity, $\dot{\theta}^{-1}(m, P, e)$. Thus, for circular orbits, the probability of observing a binary star at a given $\theta$ is uniformly distributed. For elliptical orbits, the binary star is more likely to be observed near apastron.

\subsubsection{Binary fractions}

The fraction of stars that are binaries in the systems under discussion $\left(f_{\text {bin }}\right)$ is presently entirely unconstrained, and there is some uncertainty as to the binary fraction in other stellar populations. For example, in the solar neighborhood, Duquennoy \& Mayor (1991) find an overall binary fraction of $\sim 2 / 3$ for stars near the middle of the main sequence and Hut et al. (1992) conclude that the binary fraction in globular clusters is only slightly smaller than that in the Galactic disk. Few constraints exist in dwarf galaxies; Olszewski et al. (1996) estimate a binary fraction in Draco and Ursa Minor of $0.2-0.3$ per decade in period, for periods around $1 \mathrm{yr}$, that is potentially $3-5$ times higher than in the solar neighborhood, but which is also highly uncertain. However, Lada (2006) argue that most stars are in fact single, with two-thirds of main sequence systems in the Galactic disk composed of single stars. In a small sample of globular clusters, Côté et al. (1996) have estimated a binary fraction of $\sim 0.01-0.08$ per decade of period (with significant error bars), and other studies such as Davis et al. (2008) in NGC 6397 imply a present-day binary fraction of order a percent. Formation models of globular clusters can create primordial binary fractions of $5 \%$ (Hurley et al. 2007) to $100 \%$ (Ivanova et al. 2005). In short, it is presently unclear what the primordial or current binary fraction of the systems listed in Table 1 will be.

Given the lack of constraints on $f_{b i n}$, we examine three different possible normalizations, $f_{\text {bin }}(P \leq 10 \mathrm{yrs})=0.05,0.15$ and 0.30 . The latter normalization is high, but is similar in 
magnitude to that estimated by Olszewski et al. (1996). The period distribution must be truncated at some upper limit else overall binary fractions $>1$ can be obtained depending on the shape of the assumed distribution; since we are only interested in binaries numerous enough and with short-enough periods to contribute to $\sigma_{o b s}$, we do not consider periods in excess of $10-100$ yrs.

\section{Analysis}

\subsection{Method}

We simulate four different binary populations with different assumptions about the period and eccentricity distributions: (i) log-normal in $P$, thermal in $e$; (ii) log-normal in $P, e=0$; (iii) uniform in $\log _{10} P$, thermal in $e$; (iv) uniform in $\log _{10} P, e=0$. In each case, we generate $10^{6}$ binary star systems and output the results to a file.

For each of the binary populations considered, we generate mock velocity datasets for each satellite using the following procedure:

1. For $i=1, \ldots n_{\text {stars }}$, we select an intrinsic velocity $v_{i}$ for each star from a Gaussian distribution with width $\sigma_{i n t}$;

2. For each star, we randomly designate it as single or binary according to $f_{\text {bin }}$;

3. For stars that are designated binary, we randomly assign them one of the $10^{6}$ binary orbital properties generated previously (ensuring $P_{\min } \leq P \leq P_{\max }$ ). A new value for $v_{i}$ is obtained by summing the previous value and the orbital radial velocity of the primary;

4. For each star, we assign it an observational uncertainty from the list of $n_{\text {star }}$ observational uncertainties $\left(v_{\text {err }, i}\right)$ that were actually obtained through observations 
for that satellite. These are available in the corresponding papers referenced in Table 2 or were provided by the authors (J. Simon \& M. Geha, private communication). $v_{i}$ is then modified by an error-term selected from a Gaussian distribution of width $v_{e r r, i}$;

5. Once $n_{\text {star }}$ mock radial velocities are generated according to $(1)-(4)$, we make a

first estimate of the mean $\left(\frac{\Sigma_{i}^{n_{\text {star }}} v_{i}}{n_{\text {star }}}\right)$ and standard deviation $\left(\sqrt{\frac{\sum_{i}^{n_{\text {star }}}\left(v_{i}-\bar{v}\right)^{2}}{n_{\text {star }}-1}}\right)$. All stars that are more than 3 standard deviations from the mean velocity are not considered further;

6. For the sigma-clipped sample of stars, we calculate and record the final values for the mean velocity and velocity dispersion using Equation 2;

7. Steps $(1)-(6)$ are repeated 10000 times for each satellite.

We record the percentage of times the final velocity dispersion estimate is greater than or equal to the lower $1 \sigma$ error bound of $\sigma_{o b s}$. These percentages are listed in Table 3 for each of the binary populations considered, for different values of $P_{\min }$ and $P_{\max }$, assuming different binary fractions. Figure 1 shows examples of the distribution of measured velocity dispersions from these simulations for Ursa Major I (left panel), Segue (middle panel) and Leo IV (right panel). In each panel, we show the results corresponding to $f_{\text {bin }}(P \leq 10 \mathrm{yrs})=0.15$, a log-normal binary period distribution $(1 \leq P \leq 100 \mathrm{yrs})$ and circular orbits (column 7 of Table 3).

\subsection{Results}

Table 3 shows that the binary populations considered here are very likely insufficient to explain the velocity dispersion measurements for Ursa Major I, II and Bootes I (the three systems with largest $\sigma_{o b s}$; see the left panel of Figure 1), and similarly for Coma Berenices and Canes Venatici II (unless there is a high binary fraction in these systems). 
For the remaining systems in Table 3 (Segue I, II, Willman I, Bootes II, Leo IV, V and Hercules), there is a modest, but non-negligible, chance $(\gtrsim 5 \%)$ that the measured values of $\sigma_{o b s}$ may be due to binary stars, unless the binary fraction is $\lesssim 5 \%$. For higher binary fractions, the probability that binary stars can produce $\sigma_{o b s}$ can increase to $\gtrsim 20 \%$. All of the satellites most affected have $\sigma_{o b s} \lesssim 4.5 \mathrm{~km} \mathrm{~s}^{-1}$ (with the exception of Bootes II, but here the large observational uncertainty allows a value as low as $3.1 \mathrm{~km} \mathrm{~s}^{-1}$ ). The middle panel of Figure 1 shows an example velocity dispersion distribution for Segue, a fairly typical case. Leo IV is the satellite potentially most affected by binaries (right panel of Figure 1), and inspection of Table 1 shows that this is a result of a relatively low value of $\sigma_{o b s}$ in combination with a relatively large $\tilde{\mathrm{v}}_{e r r}$.

Given that we do not know the actual binary fractions in any of the systems listed in Table 1, or the distribution of their properties, we conclude that the present observational data on the satellites with lowest $\sigma_{o b s}$ are unable to exclude at high confidence that their velocity dispersions may result from binary stars in intrinsically low-mass systems. On the other hand, $\sigma_{o b s}$ for Ursa Major I is extremely unlikely to be due to binary stars, and results for Ursa Major II, Bootes I, Coma Berenices and Canes Venatici II are unlikely to be able to be explained by binary stars unless these systems possess high binary fractions. Considered as a population, it is unlikely that all these systems have mass-to-light ratios typical of globular clusters, but boosting of the observed dispersion by binaries from near-zero values cannot be ruled out at high confidence for several individual dwarf galaxy candidates.

In line with earlier studies that dealt with higher velocity dispersion dwarfs (Mateo et al. 1993; Olszewski et al. 1996; Hargreaves et al. 1996), we find binary stars cannot boost velocity dispersions to values much in excess of $4.5 \mathrm{~km} \mathrm{~s}^{-1}$. Our results are also consistent with those of Minor et al. (2010), who examine the effect of binaries on dwarf galaxies with $\sigma_{\text {int }} \gtrsim 4 \mathrm{~km} \mathrm{~s}^{-1}$, which is an order of magnitude larger than the values of $\sigma_{\text {int }}$ considered 
here.

The binary stars that contribute to boosting the velocity dispersion from $\sigma_{\text {int }}$ to $\sigma_{\text {obs }}$ must have current orbital radial velocities of order $\sigma_{o b s}$. In our simulated data, we find that the average yearly change in the orbital radial velocities of such stars is $\sim 1-3 \mathrm{~km} \mathrm{~s}^{-1}$. To identify such stars, multi-epoch measurements (with a cadence of a year) with individual velocity uncertainties $\lesssim 1 \mathrm{~km} \mathrm{~s}^{-1}$ would be required; these observations will then directly determine the possible influence of binary stars on $\sigma_{o b s}$.

\section{Summary}

We have examined the possibility that unidentified binary stars may inflate the velocity dispersions measured for some low-mass MW satellites. If such objects were to have mass-to-light ratios similar to globular clusters, then they would be expected to have

intrinsic velocity dispersions of $0.1 \lesssim \sigma_{\text {int }} \lesssim 0.3 \mathrm{~km} \mathrm{~s}^{-1}$ - significantly below the precision of existing radial velocity measurements in these systems. Our Monte Carlo simulations reveal that, although binaries are unlikely to explain satellite velocity dispersions much in excess of $\sim 4.5 \mathrm{~km} / \mathrm{s}$, boosting of the observed dispersion by binaries cannot be ruled out with high confidence for some faint satellite candidates. Figure 2 - which plots $r_{h}$ versus $M_{V}$ for MW globular clusters, dwarf galaxies and dwarf galaxy candidates — shows that it is those dwarf candidates with $r_{h}<70 \mathrm{pc}$ (i.e. lying nearest to the globular cluster population) that are the most susceptible to contamination by spectroscopic binaries. Given the importance of obtaining accurate velocity dispersions and dynamical masses for the faintest MW satellites, it is clearly desirable to exclude directly the possible effect of binaries on these systems by obtaining multi-epoch radial velocity measurements with individual uncertainties of $\lesssim 1 \mathrm{~km} \mathrm{~s}^{-1}$. 
We thank Josh Simon and Marla Geha for sending us their data, and Peter Stetson and Nicolas Martin for a careful reading of the manuscript. 


\section{REFERENCES}

Aaronson, M. 1983, ApJ, 266, L11

Adén, D., Feltzing, S., Koch, A., Wilkinson, M. I., Grebel, E. K., Lundström, I., Gilmore, G. F., Zucker, D. B., Belokurov, V., Evans, N. W., \& Faria, D. 2009, A\&A, 506, 1147

Belokurov, V., et al. 2010, ApJ, 712, L103

Belokurov, V., Walker, M. G., Evans, N. W., Faria, D. C., Gilmore, G., Irwin, M. J., Koposov, S., Mateo, M., Olszewski, E., \& Zucker, D. B. 2008, ApJ, 686, L83

Belokurov, V., Walker, M. G., Evans, N. W., Gilmore, G., Irwin, M. J., Mateo, M., Mayer, L., Olszewski, E., Bechtold, J., \& Pickering, T. 2009, MNRAS, 397, 1748

Belokurov, V., Zucker, D. B., Evans, N. W., Kleyna, J. T., Koposov, S., Hodgkin, S. T., Irwin, M. J., Gilmore, G., Wilkinson, M. I., Fellhauer, M., Bramich, D. M., Hewett, P. C., Vidrih, S., De Jong, J. T. A., Smith, J. A., Rix, H.-W., Bell, E. F., Wyse, R. F. G., Newberg, H. J., Mayeur, P. A., Yanny, B., Rockosi, C. M., Gnedin, O. Y., Schneider, D. P., Beers, T. C., Barentine, J. C., Brewington, H., Brinkmann, J., Harvanek, M., Kleinman, S. J., Krzesinski, J., Long, D., Nitta, A., \& Snedden, S. A. 2007, ApJ, 654, 897

Belokurov, V., Zucker, D. B., Evans, N. W., Wilkinson, M. I., Irwin, M. J., Hodgkin, S., Bramich, D. M., Irwin, J. M., Gilmore, G., Willman, B., Vidrih, S., Newberg, H. J., Wyse, R. F. G., Fellhauer, M., Hewett, P. C., Cole, N., Bell, E. F., Beers, T. C., Rockosi, C. M., Yanny, B., Grebel, E. K., Schneider, D. P., Lupton, R., Barentine, J. C., Brewington, H., Brinkmann, J., Harvanek, M., Kleinman, S. J., Krzesinski, J., Long, D., Nitta, A., Smith, J. A., \& Snedden, S. A. 2006, ApJ, 647, L111 
Carlin, J. L., Grillmair, C. J., Muñoz, R. R., Nidever, D. L., \& Majewski, S. R. 2009, ApJ, 702, L9

Collins, M. L. M., et al. 2010, MNRAS, 1119

Côté, P., Pryor, C., McClure, R. D., Fletcher, J. M., \& Hesser, J. E. 1996, AJ, 112, 574

Davis, D. S., Richer, H. B., Anderson, J., Brewer, J., Hurley, J., Kalirai, J. S., Rich, R. M., \& Stetson, P. B. 2008, AJ, 135, 2155

Duquennoy, A. \& Mayor, M. 1991, A\&A, 248, 485

Geha, M., Willman, B., Simon, J. D., Strigari, L. E., Kirby, E. N., Law, D. R., \& Strader, J. 2009, ApJ, 692, 1464

Grillmair, C. J. 2009, ApJ, 693, 1118

Hargreaves, J. C., Gilmore, G., \& Annan, J. D. 1996, MNRAS, 279, 108

Harris, W. E. 1996, AJ, 112, 1487

Heggie, D. C. 1975, MNRAS, 173, 729

Hills, J. G. 1984, AJ, 89, 1811

Hurley, J. R., Aarseth, S. J., \& Shara, M. M. 2007, ApJ, 665, 707

Hut, P., et al. 1992, PASP, 104, 981

Irwin, M. J., Belokurov, V., Evans, N. W., Ryan-Weber, E. V., de Jong, J. T. A., Koposov, S., Zucker, D. B., Hodgkin, S. T., Gilmore, G., Prema, P., Hebb, L., Begum, A., Fellhauer, M., Hewett, P. C., Kennicutt, Jr., R. C., Wilkinson, M. I., Bramich, D. M., Vidrih, S., Rix, H.-W., Beers, T. C., Barentine, J. C., Brewington, H., Harvanek, M., Krzesinski, J., Long, D., Nitta, A., \& Snedden, S. A. 2007, ApJ, 656, L13 
Ivanova, N., Belczynski, K., Fregeau, J. M., \& Rasio, F. A. 2005, MNRAS, 358, 572

Kalirai, J. S., et al. 2010, ApJ, 711, 671

King, I. R. 1966, AJ, 71, 64

Koch, A., Wilkinson, M. I., Kleyna, J. T., Irwin, M., Zucker, D. B., Belokurov, V., Gilmore, G. F., Fellhauer, M., \& Evans, N. W. 2009, ApJ, 690, 453

Lada, C. J. 2006, ApJ, 640, L63

Martin, N. F., de Jong, J. T. A., \& Rix, H.-W. 2008, ApJ, 684, 1075

Martin, N. F., Ibata, R. A., Chapman, S. C., Irwin, M., \& Lewis, G. F. 2007, MNRAS, 380, 281

Mateo, M., Olszewski, E. W., Pryor, C., Welch, D. L., \& Fischer, P. 1993, AJ, 105, 510

McLaughlin, D. E. 2000, ApJ, 539, 618

Minor, Q. E., Martinez, G., Bullock, J., Kaplinghat, M., \& Trainor, R. 2010, arXiv:1001.1160

Olszewski, E. W., Pryor, C., \& Armandroff, T. E. 1996, AJ, 111, 750

Peterson, C. J. \& King, I. R. 1975, AJ, 80, 427

Pryor, C. P., Latham, D. W., \& Hazen, M. L. 1988, AJ, 96, 123

Sakamoto, T. \& Hasegawa, T. 2006, ApJ, 653, L29

Simon, J. D. \& Geha, M. 2007, ApJ, 670, 313

Walker, M. G., Belokurov, V., Evans, N. W., Irwin, M. J., Mateo, M., Olszewski, E. W., \& Gilmore, G. 2009, ApJ, 694, L144 
Walker, M. G., Mateo, M., Olszewski, E. W., Bernstein, R., Wang, X., \& Woodroofe, M. 2006, AJ, 131, 2114

Walsh, S. M., Jerjen, H., \& Willman, B. 2007, ApJ, 662, L83

Willman, B., Dalcanton, J. J., Martinez-Delgado, D., West, A. A., Blanton, M. R., Hogg, D. W., Barentine, J. C., Brewington, H. J., Harvanek, M., Kleinman, S. J., Krzesinski, J., Long, D., Neilsen, Jr., E. H., Nitta, A., \& Snedden, S. A. 2005, ApJ, 626, L85

Willman, B., et al. 2006, arXiv:astro-ph/0603486

Zucker, D. B., Belokurov, V., Evans, N. W., Kleyna, J. T., Irwin, M. J., Wilkinson, M. I., Fellhauer, M., Bramich, D. M., Gilmore, G., Newberg, H. J., Yanny, B., Smith, J. A., Hewett, P. C., Bell, E. F., Rix, H., Gnedin, O. Y., Vidrih, S., Wyse, R. F. G., Willman, B., Grebel, E. K., Schneider, D. P., Beers, T. C., Kniazev, A. Y., Barentine, J. C., Brewington, H., Brinkmann, J., Harvanek, M., Kleinman, S. J., Krzesinski, J., Long, D., Nitta, A., \& Snedden, S. A. 2006a, ApJ, 650, L41

Zucker, D. B., Belokurov, V., Evans, N. W., Wilkinson, M. I., Irwin, M. J., Sivarani, T., Hodgkin, S., Bramich, D. M., Irwin, J. M., Gilmore, G., Willman, B., Vidrih, S., Fellhauer, M., Hewett, P. C., Beers, T. C., Bell, E. F., Grebel, E. K., Schneider, D. P., Newberg, H. J., Wyse, R. F. G., Rockosi, C. M., Yanny, B., Lupton, R., Smith, J. A., Barentine, J. C., Brewington, H., Brinkmann, J., Harvanek, M., Kleinman, S. J., Krzesinski, J., Long, D., Nitta, A., \& Snedden, S. A. 2006b, ApJ, 643, L103 


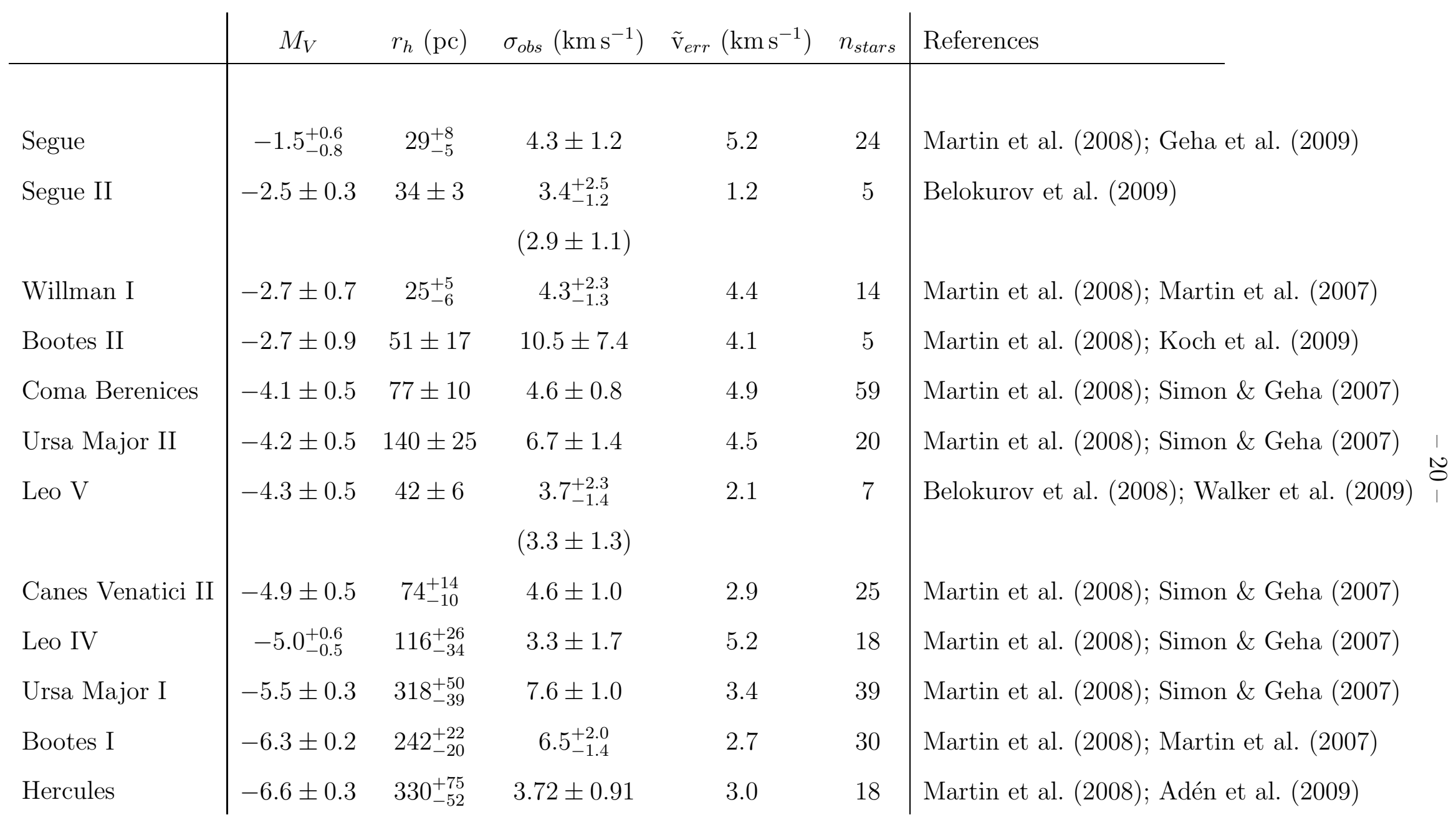

Table 1: Observed parameters and relevant kinematic data for faint MW satellites. 


\begin{tabular}{l|rrrrrrr} 
& $L_{V}$ & $c$ & $r_{c}(\mathrm{pc})$ & $\nu$ & $\alpha$ & $p$ & $\sigma_{i n t}\left(\mathrm{~km} \mathrm{~s}^{-1}\right)$ \\
\hline \multirow{2}{*}{ Segue } & & & & & & & \\
Segue II & 340 & 0.44 & 43.8 & 1.74 & 0.58 & 1.22 & $0.12 \pm 0.01$ \\
Willman I & 860 & 0.60 & 39.6 & 4.13 & 0.69 & 1.42 & $0.15 \pm 0.02$ \\
Bootes II & 1000 & 0.63 & 27.7 & 4.61 & 0.71 & 1.47 & $0.19 \pm 0.02$ \\
Coma Berenices & 1000 & 0.63 & 56.5 & 4.61 & 0.71 & 1.47 & $0.13_{-0.02}^{+0.01}$ \\
Ursa Major II & 3700 & 0.86 & 62.5 & 8.45 & 0.84 & 1.70 & $0.21 \pm 0.02$ \\
Leo V & 4100 & 0.87 & 111.3 & 8.75 & 0.84 & 1.71 & $0.16 \pm 0.02$ \\
Canes Venatici II & 7500 & 0.89 & 32.7 & 9.06 & 0.85 & 1.72 & $0.31 \pm 0.03$ \\
Leo IV & 7800 & 0.99 & 51.3 & 10.95 & 0.87 & 1.78 & $0.31 \pm 0.03$ \\
Ursa Major I & 8600 & 1.00 & 78.8 & 11.27 & 0.88 & 1.78 & $0.26 \pm 0.03$ \\
Bootes I & 14000 & 1.08 & 196.8 & 12.95 & 0.90 & 1.82 & $0.19 \pm 0.02$ \\
Hercules & 28000 & 1.21 & 128.9 & 15.84 & 0.92 & 1.86 & $0.32_{-0.04}^{+0.03}$ \\
\hline
\end{tabular}

Table 2: Derived characteristics of faint MW satellites assuming $\Upsilon_{V}=1.45$. 


\begin{tabular}{|c|c|c|c|c|c|c|c|c|c|c|c|c|c|c|c|c|}
\hline \multirow{3}{*}{$\begin{array}{l}P_{\min }(\mathrm{yrs}) \\
P_{\max }(\mathrm{yrs})\end{array}$} & \multicolumn{4}{|c|}{$\begin{array}{c}\log _{10} P \text { (normal) } \\
e(\text { thermal })\end{array}$} & \multicolumn{4}{|c|}{$\begin{array}{c}\log _{10} P \text { (normal) } \\
e \text { (circular) }\end{array}$} & \multicolumn{4}{|c|}{$\begin{array}{c}\log _{10} P \text { (uniform) } \\
e(\text { thermal })\end{array}$} & \multicolumn{4}{|c|}{$\begin{array}{c}\log _{10} P \text { (uniform) } \\
e \text { (circular) }\end{array}$} \\
\hline & 1 & 0.1 & 1 & 0.1 & 1 & 0.1 & 1 & 0.1 & 1 & 0.1 & 1 & 0.1 & 1 & 0.1 & 1 & 0.1 \\
\hline & 10 & 10 & 100 & 100 & 10 & 10 & 100 & 100 & 10 & 10 & 100 & 100 & 10 & 10 & 100 & 100 \\
\hline & \multicolumn{16}{|c|}{$f_{\text {bin }}(P \leq 10 \mathrm{yrs})=5 \%$} \\
\hline Segue & 3.6 & 5.6 & 3.9 & 5.5 & 4.1 & 6.5 & 4.2 & 6.8 & 3.6 & 5.9 & 4.1 & 6.3 & 3.8 & 8.3 & 4.0 & 8.3 \\
\hline Segue II & 4.8 & 7.2 & 6.4 & 7.0 & 6.4 & 8.1 & 7.8 & 9.2 & 4.8 & 7.3 & 6.4 & 7.7 & 6.1 & 10.1 & 8.4 & 10.0 \\
\hline Willman I & 2.4 & 4.3 & 2.9 & 4.4 & 2.9 & 5.2 & 3.4 & 5.8 & 2.5 & 5.3 & 3.0 & 5.2 & 2.9 & 6.7 & 3.0 & 6.4 \\
\hline Bootes II & 4.7 & 5.8 & 5.1 & 6.3 & 5.0 & 6.3 & 5.6 & 6.8 & 5.0 & 6.4 & 5.2 & 6.5 & 4.7 & 6.9 & 5.6 & 7.0 \\
\hline Coma Berenices & 0.3 & 1.3 & 0.4 & 1.2 & 0.1 & 1.5 & 0.0 & 1.6 & 0.2 & 1.9 & 0.4 & 1.8 & 0.1 & 2.4 & 0.1 & 2.4 \\
\hline Ursa Major II & 0.2 & 0.8 & 0.4 & 0.7 & 0.1 & 1.0 & 0.1 & 0.9 & 0.2 & 0.9 & 0.2 & 1.3 & 0.0 & 1.1 & 0.1 & 1.2 \\
\hline Leo V & 5.5 & 8.0 & 6.7 & 9.0 & 6.8 & 10.0 & 8.7 & 10.6 & 6.2 & 8.7 & 6.9 & 9.4 & 7.1 & 10.7 & 7.7 & 11.0 \\
\hline Canes Venatici II & 0.5 & 1.8 & 0.7 & 1.8 & 0.3 & 2.2 & 0.5 & 2.3 & 0.5 & 2.4 & 0.6 & 2.0 & 0.3 & 3.2 & 0.4 & 3.1 \\
\hline Leo IV & 21.4 & 24.4 & 23.5 & 24.9 & 22.9 & 26.0 & 24.5 & 27.1 & 22.3 & 25.0 & 23.0 & 25.3 & 23.1 & 27.7 & 23.9 & 27.3 \\
\hline Ursa Major I & 0.0 & 0.0 & 0.0 & 0.0 & 0.0 & 0.0 & 0.0 & 0.0 & 0.0 & 0.0 & 0.0 & 0.0 & 0.0 & 0.0 & 0.0 & 0.0 \\
\hline Bootes I & 0.0 & 0.2 & 0.0 & 0.2 & 0.0 & 0.2 & 0.0 & 0.2 & 0.0 & 0.1 & 0.1 & 0.2 & 0.0 & 0.3 & 0.0 & 0.2 \\
\hline \multirow[t]{2}{*}{ Hercules } & 1.4 & 3.2 & 1.8 & 3.2 & 1.5 & 4.6 & 2.0 & 4.6 & 1.7 & 3.4 & 1.8 & 3.8 & 2.0 & 4.9 & 1.9 & 5.4 \\
\hline & \multicolumn{16}{|c|}{$f_{\text {bin }}(P \leq 10 \mathrm{yrs})=15 \%$} \\
\hline Segue & 7.7 & 14.1 & 8.8 & 15.4 & 8.6 & 18.0 & 10.4 & 19.7 & 8.6 & 15.8 & 9.7 & 17.4 & 9.4 & 21.5 & 10.5 & 22.1 \\
\hline Segue II & 13.0 & 18.8 & 17.0 & 21.3 & 17.2 & 23.7 & 21.4 & 25.4 & 13.2 & 19.6 & 16.6 & 21.2 & 17.5 & 25.4 & 21.0 & 27.7 \\
\hline Willman I & 6.8 & 12.0 & 8.4 & 12.8 & 7.6 & 16.6 & 9.4 & 16.5 & 6.7 & 14.3 & 8.4 & 14.6 & 8.6 & 18.9 & 9.3 & 19.1 \\
\hline Bootes II & 8.0 & 11.1 & 8.6 & 11.8 & 8.4 & 13.4 & 9.6 & 14.5 & 7.6 & 12.0 & 8.9 & 12.2 & 8.8 & 14.8 & 9.5 & 15.2 \\
\hline Coma Berenices & 1.2 & 5.8 & 1.7 & 6.0 & 0.5 & 7.9 & 0.6 & 8.4 & 1.5 & 7.8 & 1.7 & 8.3 & 0.6 & 11.6 & 0.6 & 11.9 \\
\hline Ursa Major II & 0.7 & 2.9 & 0.8 & 2.9 & 0.1 & 3.1 & 0.2 & 3.6 & 0.5 & 3.9 & 0.6 & 3.9 & 0.1 & 4.6 & 0.2 & 4.9 \\
\hline Leo V & 13.0 & 19.8 & 16.6 & 22.2 & 17.3 & 24.8 & 21.3 & 27.4 & 13.5 & 21.6 & 17.6 & 23.9 & 16.7 & 28.4 & 21.6 & 30.3 \\
\hline Canes Venatici II & 2.3 & 7.2 & 2.9 & 7.6 & 1.3 & 9.7 & 2.3 & 11.3 & 2.5 & 8.8 & 2.7 & 9.1 & 1.8 & 13.3 & 2.3 & 14.0 \\
\hline Leo IV & 29.4 & 36.7 & 33.7 & 38.7 & 31.9 & 41.1 & 37.1 & 43.0 & 29.6 & 38.8 & 32.3 & 38.7 & 32.0 & 43.4 & 36.3 & 45.1 \\
\hline Ursa Major I & 0.0 & 0.1 & 0.0 & 0.1 & 0.0 & 0.1 & 0.0 & 0.1 & 0.0 & 0.2 & 0.0 & 0.2 & 0.0 & 0.2 & 0.0 & 0.1 \\
\hline Bootes I & 0.1 & 0.9 & 0.2 & 1.2 & 0.0 & 1.3 & 0.0 & 1.5 & 0.2 & 1.5 & 0.2 & 1.6 & 0.0 & 2.3 & 0.0 & 2.6 \\
\hline \multirow[t]{2}{*}{ Hercules } & 4.9 & 11.1 & 7.4 & 12.8 & 7.3 & 16.1 & 9.2 & 17.9 & 5.4 & 13.5 & 7.5 & 13.9 & 7.5 & 20.0 & 8.5 & 20.8 \\
\hline & \multicolumn{16}{|c|}{$f_{\text {bin }}(P \leq 10$ yrs $)=30 \%$} \\
\hline Segue & 14.7 & 27.9 & 18.7 & 30.9 & 17.8 & 37.2 & 22.6 & 40.0 & 16.2 & 33.0 & 19.4 & 34.6 & 18.2 & 43.1 & 22.8 & 43.6 \\
\hline Segue II & 24.4 & 34.0 & 33.9 & 39.4 & 33.3 & 43.2 & 41.9 & 46.8 & 25.5 & 37.0 & 31.6 & 40.7 & 31.3 & 45.7 & 40.3 & 48.7 \\
\hline Willman I & 12.9 & 24.8 & 16.5 & 26.1 & 15.5 & 30.8 & 19.7 & 34.0 & 14.0 & 27.7 & 16.9 & 29.5 & 16.5 & 37.5 & 20.7 & 38.2 \\
\hline Bootes II & 13.0 & 18.6 & 14.9 & 19.6 & 14.0 & 23.3 & 16.6 & 24.2 & 12.0 & 20.6 & 13.9 & 21.8 & 14.3 & 25.4 & 16.2 & 27.3 \\
\hline Coma Berenices & 4.2 & 17.6 & 6.1 & 19.2 & 2.5 & 26.3 & 4.7 & 28.3 & 4.7 & 22.6 & 6.0 & 24.4 & 2.8 & 34.9 & 4.5 & 36.8 \\
\hline Ursa Major II & 1.7 & 7.1 & 2.2 & 7.5 & 0.7 & 9.0 & 0.8 & 10.0 & 1.8 & 9.6 & 2.1 & 9.7 & 0.6 & 12.8 & 0.8 & 12.9 \\
\hline Leo V & 24.5 & 36.0 & 31.9 & 40.8 & 31.6 & 44.1 & 41.3 & 49.7 & 26.1 & 40.0 & 32.0 & 43.7 & 31.5 & 48.6 & 39.7 & 52.1 \\
\hline Canes Venatici II & 6.3 & 18.9 & 9.1 & 21.5 & 6.4 & 27.1 & 8.8 & 30.4 & 6.8 & 23.3 & 8.7 & 24.2 & 6.2 & 35.1 & 9.0 & 36.5 \\
\hline Leo IV & 40.4 & 52.4 & 47.3 & 54.7 & 44.4 & 58.8 & 52.7 & 63.2 & 41.2 & 55.2 & 45.0 & 57.9 & 45.2 & 63.3 & 51.2 & 65.2 \\
\hline Ursa Major I & 0.0 & 0.5 & 0.0 & 0.5 & 0.0 & 0.4 & 0.0 & 0.7 & 0.0 & 1.1 & 0.0 & 1.1 & 0.0 & 1.1 & 0.0 & 1.2 \\
\hline Bootes I & 0.6 & 3.9 & 0.9 & 4.5 & 0.1 & 6.4 & 0.1 & 7.4 & 0.6 & 6.0 & 0.6 & 6.2 & 0.1 & 10.0 & 0.1 & 10.8 \\
\hline Hercules & 12.8 & 25.5 & 19.8 & 31.1 & 18.6 & 38.7 & 25.4 & 42.1 & 14.1 & 31.7 & 19.1 & 33.1 & 19.2 & 44.1 & 24.8 & 46.9 \\
\hline
\end{tabular}

Table 3: Simulation results for each satellite assuming $f_{\text {bin }}(P \leq 10 \mathrm{yrs})=5 \%, 15 \%$ and $30 \%$ (upper, middle and lower sections, respectively), for different period and eccentricity assumptions. Numbers refer to the percentage of simulations that generate a velocity dispersion that is greater than or equal to the $1 \sigma$ lower error-bound on the observed velocity dispersion. 

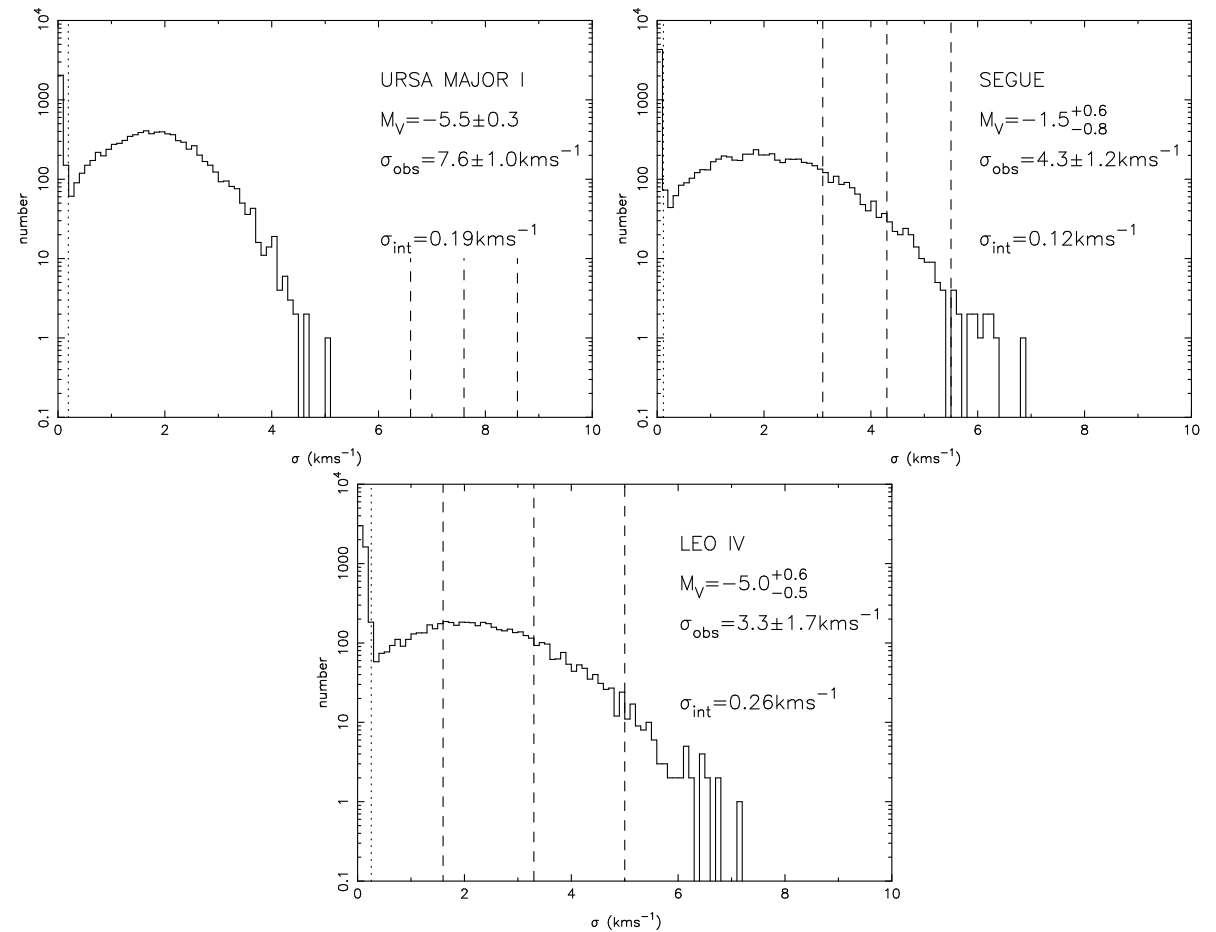

Fig. 1.- The simulated velocity dispersion distribution for Ursa Major I, Segue, and Leo IV, assuming $f_{\text {bin }}(P \leq 10 \mathrm{yrs})=15 \%$, a log-normal period distribution with $1 \leq P \leq 100$ yrs and circular orbits (column 7 of Table 3 ). The dotted line shows the assumed intrinsic velocity dispersion, and the dashed lines show the observed velocity dispersion with $1 \sigma$ error bounds. Ursa Major I is incompatible with an intrinsically low mass system, but this hypothesis cannot be excluded at high confidence for Segue and Leo IV. 


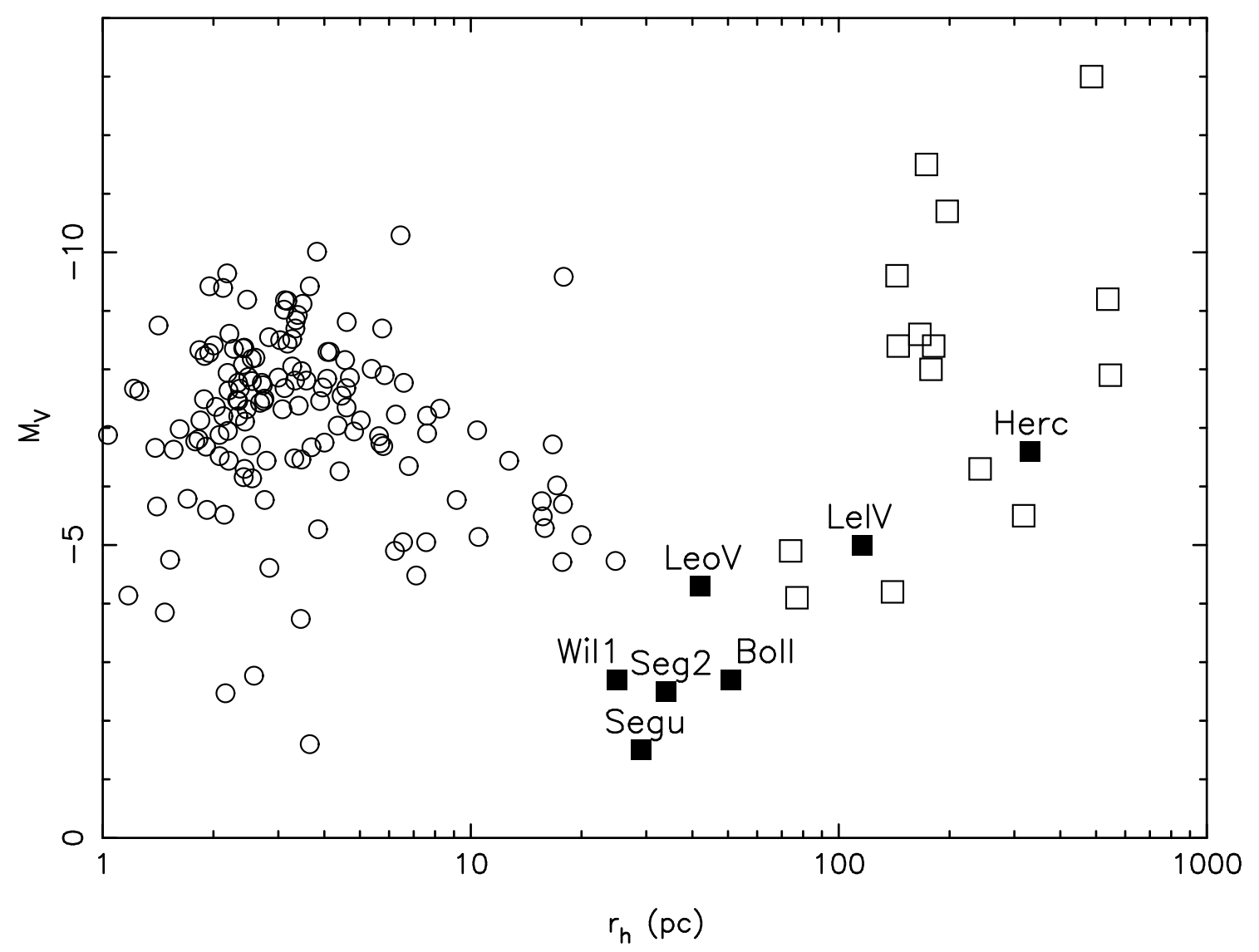

Fig. 2. - $r_{h}$ versus $M_{V}$ for MW satellites. Open circles correspond to globular clusters (Harris 1996), squares correspond to dwarf galaxies and dwarf candidates. The putative dwarfs whose velocity dispersions are most susceptible to boosting by spectroscopic binaries are shown as filled squares. These include all dwarf candidates with $r_{h} \lesssim 70 \mathrm{pc}$ that lie closest to the globular cluster population. 